\title{
Three-species competition with non-deterministic outcomes
}

\author{
Tim M. A. Depraetere, ${ }^{\text {a) }}$ Aisling J. Daly, Jan M. Baetens, and Bernard De Baets \\ KERMIT, Department of Data Analysis and Mathematical Modelling, Ghent University, Coupure links 653, \\ B-9000 Ghent, Belgium
}

(Received 2 July 2018; accepted 3 December 2018; published online 27 December 2018)

\begin{abstract}
Theoretical and experimental research studies have shown that ecosystems governed by nontransitive competition networks tend to maintain high levels of biodiversity. The theoretical body of work, however, has mainly focused on competition networks in which the outcomes of competition events are predetermined and hence deterministic, and where all species are identical up to their competitive relationships, an assumption that may limit the applicability of theoretical results to real-life situations. In this paper, we aim to probe the robustness of the link between biodiversity and non-transitive competition by introducing a three-dimensional winning probability parameter space, making the outcomes of competition events in a three-species in silico ecosystem uncertain. While two degenerate points in this parameter space have been the subject of previous studies, we investigate the remaining settings, which equip the species with distinct competitive abilities. We find that the impact of this modification depends on the spatial dimension of the system. When the system is well mixed, it collapses to monoculture, as is also the case in the non-transitive deterministic setting. In one dimension, chaotic patterns emerge, which tend to maintain biodiversity, and a power law relates the time that species manage to coexist to the degree of uncertainty regarding competition event outcomes. In two dimensions, the formation of spiral wave patterns ensures that biodiversity is maintained for moderate degrees of uncertainty, while considerable deviations from the non-transitive deterministic setting have strong negative effects on species coexistence. It can hence be concluded that non-transitive competition can still produce coexistence when the assumption of deterministic competition is abandoned. When the system collapses to monoculture, one observes a "survival of the strongest" law, as the species that has the highest probability of defeating its competitors has the best odds to become the sole survivor. Published by AIP Publishing. https://doi.org/10.1063/1.5046795
\end{abstract}

One of the main enigmas in ecology concerns biodiversity is: How do species manage to coexist? The intricate structures found in ecological interaction networks, i.e., graphs summarizing competitive, mutualistic, predatory, or parasitic relationships among species, have been pinpointed as a possible answer to the biodiversity conundrum, as they allow for the coexistence of a large variety of species. In particular, the merits of cyclic competition as a mechanism supporting coexistence have been studied extensively from a theoretical and experimental point of view. Species constituting a community governed by such a cyclic competition network compete with each other much like rock, paper, and scissors do in the popular children's game. Theoretical research has shown that this behavior results in the formation of spatial patterns, which allows all species to coexist. These findings, however, are based on the assumption that the outcome of a competition event is predetermined and hence deterministic, as such neglecting intraspecific variability and the fact that a competitive advantage depends on fluctuating environmental conditions, while at the same time mandating that all species are identical up to their competitive relationships. In this paper, by means of individual-based models and mean-field equations, we study whether cyclic competition can still result in coexistence when this idealization is abandoned. It was found that results depend on the spatial

\footnotetext{
a)Electronic mail: tim.depraetere@ugent.be
}

dimension of the system, and the degree of uncertainty regarding the competition event outcomes. When the system is well mixed, two out of three species go extinct. In one spatial dimension, the time that species manage to coexist is related to the degree of uncertainty by a power law, partly due to the formation of chaotic spatial patterns. In two spatial dimensions, up to moderate degrees of uncertainty, the species organise themselves in spiral wave patterns allowing for coexistence, while higher degrees of uncertainty produce a monoculture. Hence, in general, cyclic competition can still maintain biodiversity, as long as the degree of uncertainty regarding the competition event outcomes is not too high. In the case that the dynamics produce a monoculture, a "survival of the strongest" law can be discerned, as the species that has the highest probability of defeating its competitors has the best odds to become the sole survivor.

\section{INTRODUCTION}

Gause's competitive exclusion principle states that when several species compete for one limiting resource, only the fittest one among them survives. ${ }^{1,2}$ However, natural ecosystems support more species than the principle predicts, as is strikingly illustrated by the paradox of the plankton. ${ }^{3,4}$ In order to elucidate this biodiversity enigma, chaotic population dynamics, ${ }^{5,6}$ co-evolution, ${ }^{7}$ spatial heterogeneity, ${ }^{8}$ and 
the structure of ecological interaction networks ${ }^{9,10}$ have been considered, amongst others.

A subset of biodiversity research on ecological interaction networks focuses on transitivity in tournament graphs which summarize competitive interactions among species, ${ }^{11-34}$ hereafter referred to as competition networks. When three species are involved, there exist two such nonisomorphic competition networks. On the one hand, in the non-transitive competition network, displayed in Fig. 1(a), the interactions between species are organized in a cyclic manner, in the sense that A dominates B, while B dominates $\mathrm{C}$, and $\mathrm{C}$ in its turn dominates $\mathrm{A}$. Theoretical studies on in silico ecosystems show that such a non-transitive competition network, combined with the dispersal of species through space, results in long-term conservation of biodiversity through the formation of spatial patterns. ${ }^{16-18,35-38}$ On the other hand, in the transitive competition network, displayed in Fig. 1(c), the species dominance relationships are organized in a hierarchical manner, which results in monoculture through the extinction of all but the most dominant species.

Theoretical research in the area of non-transitive competition has been complemented by the discovery of such competition networks in a variety of real-world ecosystems. Examples include coral reef invertebrates, side-blotched lizards in California, and lemmings in Greenland. ${ }^{39-41}$ Moreover, in vitro experiments have been conducted in which colicin-producing, -sensitive and -resistant E. coli strains compete in a non-transitive manner, the results of which seem to indicate that coexistence is maintained through the spatial segregation of the strains..$^{37,42}$ However, more thorough studies are needed in order to conclude whether non-transitive competition actually plays a role in real-life biodiversity maintenance. ${ }^{43}$

The competition networks in Figs. 1(a) and 1(c) are deterministic in the sense that a competition event involving species A and B is always won by species A, and so forth. At least two problems can be identified with this conceptualization. First, in nature, the outcome of competition events is dependent on fluctuating environmental conditions. Hunting success of lions, for instance, depends on wind direction, moon brightness, and grass height, ${ }^{44}$ while fluctuations in light intensity in the ocean influence competition in phytoplankton communities. ${ }^{45}$ Secondly, there is intraspecific variability, so that the competitive abilities of individuals belonging to the same species can differ. Both these arguments imply that, in reality, competitive dominance is less pronounced than the deterministic competition networks propose, ${ }^{27-29,46-48}$ to

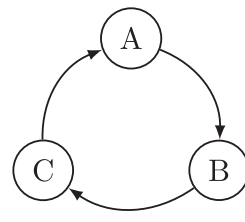

(a)

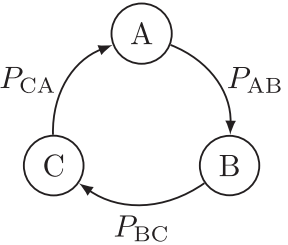

(b)

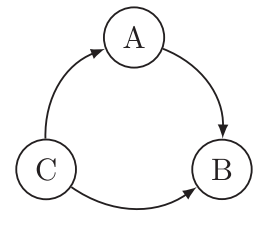

(c)
FIG. 1. An ecosystem consisting of species A, B, and C governed by a non-transitive deterministic (a), a non-deterministic (b), and a transitive deterministic (c) competition network. the extent that it may not occur in reality. Moreover, the determinism entails that each cyclic permutation of the vertices $\mathrm{A}, \mathrm{B}$, and $\mathrm{C}$ is an automorphism of the non-transitive deterministic competition network. While endowing an in silico ecosystem with such symmetry could be considered as unnatural and artificial, it has been shown to be the crucial ingredient for species to coexist in a well-mixed setting. ${ }^{15}$

In order to make the deterministic competition networks more realistic, we introduce winning probabilities $P_{\mathrm{AB}}, P_{\mathrm{BC}}$, $P_{\mathrm{CA}} \in[0,1]$, expressing that the outcomes of competition events are uncertain. Their interpretation is as follows: when an individual of species $A$ competes with an individual of species $\mathrm{B}$, A dominates $\mathrm{B}$ with probability $P_{\mathrm{AB}}$, or is dominated by $\mathrm{B}$ with probability $P_{\mathrm{BA}} \equiv 1-P_{\mathrm{AB}}$, and so forth. The resulting non-deterministic competition network is displayed in Fig. 1(b).

Variation of the winning probabilities can take the competition network from the non-transitive deterministic setting which has been the subject of previous studies ${ }^{13-15,17-20,23,26,28,31-34}$ [Fig. 1(a)], when $P_{\mathrm{AB}}=P_{\mathrm{BC}}=$ $P_{\mathrm{CA}}=1$ or $P_{\mathrm{AB}}=P_{\mathrm{BC}}=P_{\mathrm{CA}}=0$, to the transitive deterministic setting [e.g., Fig. 1(c)], when either one or two of the winning probabilities equal zero while the other(s) equal(s) one. The non-deterministic settings in between those extremes can be subdivided in weakly stochastic transitive competition networks, for which it holds that $\left(P_{i j} \geq 1 / 2 \wedge P_{j k} \geq 1 / 2\right) \Rightarrow P_{k i} \leq 1 / 2$, for any $(i, j, k) \in$ $\{\mathrm{A}, \mathrm{B}, \mathrm{C}\}^{3}$, and not weakly stochastic transitive competition networks. ${ }^{49}$

Unequal winning probabilities can endow the species with distinct competitive abilities, as such breaking the symmetry associated with the non-transitive deterministic competition network. When $P_{\mathrm{AB}} \neq P_{\mathrm{BC}}$, the rate at which $\mathrm{A}$ outcompetes $\mathrm{B}$ differs from the rate at which $\mathrm{B}$ outcompetes C. Investigating coexistence under such unequal competition rates is of particular interest, as it was shown recently that in the E. coli system mentioned previously, ${ }^{37}$ the colicinproducing strain outcompetes the sensitive strain at a relatively high rate compared to the rates related to competition events involving the resistant strain. ${ }^{50}$

In this paper, we show how the population dynamics emerging from non-transitive competition among three species is affected by the introduction of winning probabilities. In Sec. II, we introduce the individual-based model used to describe the dynamics of an ecosystem governed by this competition network, while in Secs. III-V, we study the dynamics of this model in a well-mixed setting, and in one and two spatial dimensions. Real-life counterparts of these habitats are laboratory flasks with mixing equipment, quasione-dimensional channels, ${ }^{51}$ and Petri dishes,${ }^{37}$ respectively. In Sec. VI, we summarize our findings in terms of their effects on coexistence.

\section{AN INDIVIDUAL-BASED MODEL}

The biological processes driving population dynamics, such as competition, reproduction, and diffusion, are generally considered to be of a stochastic nature, and to depend on 
the spatial distribution of species. ${ }^{52}$ For these reasons, population dynamics are often modelled using individual-based models (IBMs). These interacting particle systems, in which time, space, and state are discrete, treat space explicitly. ${ }^{53}$ Their dynamics are determined by game-theoretical rules that dictate the outcomes of the interactions among individuals, and are thus emergent.

Usually, three such rules are defined to represent competition, reproduction, and exchange events, in order to make in silico ecosystems mimic their real-life counterparts. ${ }^{19,20,54}$ This set of rules constitutes the May-Leonard model. ${ }^{15}$ In the context of non-deterministic competition, the competition rules are given by

$$
\begin{array}{ll}
\mathrm{AB} \stackrel{P_{\mathrm{AB} \sigma}}{\longrightarrow} \mathrm{A} \oslash, & \mathrm{AB} \stackrel{\left(1-P_{\mathrm{AB}}\right) \sigma}{\longrightarrow} \oslash \mathrm{B}, \\
\mathrm{BC} \stackrel{P_{\mathrm{BC}} \sigma}{\longrightarrow} \mathrm{B} \oslash, & \mathrm{BC} \stackrel{\left(1-P_{\mathrm{BC}}\right) \sigma}{\longrightarrow} \oslash \mathrm{C}, \\
\mathrm{CA} \stackrel{P_{\mathrm{CA}} \sigma}{\longrightarrow} \mathrm{C} \oslash, & \mathrm{CA} \stackrel{\left(1-P_{\mathrm{CA}}\right) \sigma}{\longrightarrow} \oslash \mathrm{A} .
\end{array}
$$

One thus observes that when two individuals belonging to species $A$ and $B$ engage in a competition event, the outcome is either the death of individual $\mathrm{B}$ with probability $P_{\mathrm{AB}}$ or the death of individual A with probability $1-P_{\mathrm{AB}}$, as prescribed by the competition network in Fig. 1(b). The space that was occupied by the killed individual becomes empty $(\oslash)$. A positive reaction rate constant, $\sigma$, is associated with the competition rules, whose meaning will become clear upon introducing the algorithm that evolves the IBM.

The rules encoding reproduction events are given by

$$
\mathrm{X} \oslash \stackrel{\mu}{\rightarrow} \mathrm{XX},
$$

with $\mathrm{X} \in\{\mathrm{A}, \mathrm{B}, \mathrm{C}\}$, and $\mu>0$ a reaction rate constant associated with reproduction, while the rules encoding exchange events are

$$
\mathrm{YZ} \stackrel{\epsilon}{\rightarrow} \mathrm{ZY},
$$

with $\mathrm{Y}, \mathrm{Z} \in\{\mathrm{A}, \mathrm{B}, \mathrm{C}, \oslash\}$, and $\epsilon>0$ the corresponding reaction rate constant.

With everything in place, we now outline the algorithm that incorporates the game-theoretical rules and builds them into an IBM. For this purpose, we consider a $d$-dimensional space subject to periodic boundary conditions, and divide it into $N^{d}$ identical hypercubes, hereafter referred to as cells. As an initial condition, every cell is randomly assigned either an individual of a certain species (A, B, or C) or is left empty, each of these four scenarios occurring with probability $1 / 4$, unless stated otherwise. During one step of the algorithm, a randomly selected cell interacts with another randomly selected cell in its von Neumann neighbourhood. A random number determines the interaction that occurs: a reproduction event, with probability $\mu$, a competition event, with probability $\sigma$, or an exchange event, with probability $\epsilon$. Note that this requires $\mu+\sigma+\epsilon \leq 1$ when setting the reaction rate constants. If the contents of both selected cells allow for it, the chosen event takes place, and the state of the system is updated accordingly. In either case, time is advanced by one unit. In order to comply with the literature, we define a generation as $N^{d}$ units of time, such that a generation is the time necessary for each cell, on average, to be involved in two interaction events. Note that during a competition event, in a system governed by a non-deterministic competition network, an additional random number is drawn and compared to the appropriate winning probability to determine the victor of the event.

In the subsequent sections, we study the dynamics of this IBM in a well-mixed setting, and in one and two spatial dimensions, in terms of the coexistence time, i.e., the number of generations until one of the species goes extinct, and the sole survivor, i.e., the species still present in the system after the other two have gone extinct. To understand how the nondeterministic competition network changes the dynamics of the in silico ecosystem, we will study the dynamics for varying winning probabilities, while the other system parameters are kept constant.

\section{THE WELL-MIXED SYSTEM}

In a perfectly well-mixed setting, all individuals in the system can interact with one another. The IBM described in Sec. II remains valid, but the neighbourhood of a cell now constitutes the whole system, such that space becomes irrelevant. We define the system to consist out of $N$ cells, and, hence, a generation to consist out of $N$ simulation steps.

\section{A. Dynamics in the limit $N \rightarrow \infty$}

In order to study this system, we approximate the dynamics of the IBM by a set of ordinary differential equations (ODEs) through a van Kampen expansion, a procedure that becomes exact in the limit $N \rightarrow \infty .{ }^{55}$ This results in the following system of ODEs:

$$
\left\{\begin{array}{l}
\dot{a}=a(1-\rho)-k a\left[P_{\mathrm{CA}} c+\left(1-P_{\mathrm{AB}}\right) b\right] \equiv f_{1}, \\
\dot{b}=b(1-\rho)-k b\left[P_{\mathrm{AB}} a+\left(1-P_{\mathrm{BC}}\right) c\right] \equiv f_{2}, \\
\dot{c}=c(1-\rho)-k c\left[P_{\mathrm{BC}} b+\left(1-P_{\mathrm{CA}}\right) a\right] \equiv f_{3} .
\end{array}\right.
$$

Here, $a, b$, and $c$ represent the densities of species A, B, and C, respectively, $\rho \equiv a+b+c$ and $k \equiv \sigma / \mu$. Since we wish to consider the setting where both competition and reproduction play a role, we choose $k>0$. In biological systems, negative population densities are not allowed, so we restrict to $(a, b, c) \in \mathbb{R}_{+}^{3} \equiv\left\{(a, b, c) \in \mathbb{R}^{3} \mid a, b, c \geq 0\right\}$. Note that solutions with initial conditions in $\mathbb{R}_{+}^{3}$ do not leave $\mathbb{R}_{+}^{3}$. We now study the dynamics of this system for $\left.P_{\mathrm{AB}}, P_{\mathrm{BC}}, P_{\mathrm{CA}} \in\right] 0,1[$.

System (4) belongs to the class of competitive Lotka-Volterra equations involving three species, which has been investigated in Ref. 56. We now apply the results from Ref. 56 to our system governed by non-deterministic competition.

The origin, $\mathbf{x}_{1}^{*}=(0,0,0)$, is an unstable fixed point for all considered parameter values. The boundary of the basin of repulsion of the origin in $\mathbb{R}_{+}^{3}$ is called the carrying simplex. It has been shown in Ref. 57 that all fixed points and other limit sets of System (4), except the origin, lie on this carrying simplex. Supported by these findings, the analysis of the dynamics of System (4) can be restricted to the dynamics on the carrying simplex. Moreover, it has been shown that no limit cycles are present in System (4), under the parameter range considered in this paper. ${ }^{58}$ 
The carrying simplex can contain up to seven fixed points. Three of them correspond to the survival of one species

$$
\begin{aligned}
& \mathbf{x}_{2}^{*}=(1,0,0), \\
& \mathbf{x}_{3}^{*}=(0,1,0), \\
& \mathbf{x}_{4}^{*}=(0,0,1),
\end{aligned}
$$

while another three indicate the survival of two species

$$
\begin{aligned}
& \mathbf{x}_{5}^{*}=\left(\frac{1-P_{\mathrm{AB}}}{1+P_{\mathrm{AB}}\left(1-P_{\mathrm{AB}}\right)}, \frac{P_{\mathrm{AB}}}{1+P_{\mathrm{AB}}\left(1-P_{\mathrm{AB}}\right)}, 0\right), \\
& \mathbf{x}_{6}^{*}=\left(\frac{P_{\mathrm{CA}}}{1+P_{\mathrm{CA}}\left(1-P_{\mathrm{CA}}\right)}, 0, \frac{1-P_{\mathrm{CA}}}{1+P_{\mathrm{CA}}\left(1-P_{\mathrm{CA}}\right)}\right), \\
& \mathbf{x}_{7}^{*}=\left(0, \frac{1-P_{\mathrm{BC}}}{1+P_{\mathrm{BC}}\left(1-P_{\mathrm{BC}}\right)}, \frac{P_{\mathrm{BC}}}{1+P_{\mathrm{BC}}\left(1-P_{\mathrm{BC}}\right)}\right),
\end{aligned}
$$

and, finally, there is one fixed point representing the coexistence of three species

$$
\mathbf{x}_{8}^{*}=\frac{1}{\alpha}\left(a_{8}^{*}, b_{8}^{*}, c_{8}^{*}\right),
$$

where

$$
\begin{aligned}
\alpha= & P_{\mathrm{AB}}^{2}+P_{\mathrm{BC}}^{2}+\left(P_{\mathrm{CA}}-2\right)^{2}+P_{\mathrm{BC}}\left(3 P_{\mathrm{CA}}-4\right) \\
& +P_{\mathrm{AB}}\left(3 P_{\mathrm{CA}}+3 P_{\mathrm{BC}}-4\right), \\
a_{8}^{*}= & P_{\mathrm{BC}}^{2}+P_{\mathrm{BC}}\left(P_{\mathrm{CA}}+P_{\mathrm{AB}}-2\right)-P_{\mathrm{AB}}+1, \\
b_{8}^{*}= & P_{\mathrm{CA}}^{2}+P_{\mathrm{CA}}\left(P_{\mathrm{BC}}+P_{\mathrm{AB}}-2\right)-P_{\mathrm{BC}}+1, \\
c_{8}^{*}= & P_{\mathrm{AB}}^{2}+P_{\mathrm{AB}}\left(P_{\mathrm{CA}}+P_{\mathrm{BC}}-2\right)-P_{\mathrm{CA}}+1 .
\end{aligned}
$$

Fixed points $\mathbf{x}_{2}^{*}, \mathbf{x}_{3}^{*}$, and $\mathbf{x}_{4}^{*}$ are asymptotically stable for all parameter values considered. In order to classify the stability of the other fixed points, we distinguish between settings for which the following three conditions are not satisfied, and settings for which they are:

$$
\begin{aligned}
& P_{\mathrm{CA}}<-\frac{\left(P_{\mathrm{BC}}-1\right)\left(P_{\mathrm{AB}}+P_{\mathrm{BC}}-1\right)}{P_{\mathrm{BC}}} \\
& \wedge P_{\mathrm{AB}}>1-P_{\mathrm{BC}}, \\
& P_{\mathrm{AB}}<2-2 \sqrt{1-P_{\mathrm{BC}}}-P_{\mathrm{BC}} \\
& \wedge P_{\mathrm{CA}}> \frac{1}{2}\left(2-P_{\mathrm{AB}}-P_{\mathrm{BC}}\right. \\
&\left.-\sqrt{P_{\mathrm{AB}}^{2}+2 P_{\mathrm{AB}}\left(P_{\mathrm{BC}}-2\right)+P_{\mathrm{BC}}^{2}}\right) \\
& \wedge P_{\mathrm{CA}}< \frac{1}{2}\left(2-P_{\mathrm{AB}}-P_{\mathrm{BC}}\right. \\
&\left.+\sqrt{P_{\mathrm{AB}}^{2}+2 P_{\mathrm{AB}}\left(P_{\mathrm{BC}}-2\right)+P_{\mathrm{BC}}^{2}}\right), \\
& P_{\mathrm{CA}}>1-\frac{P_{\mathrm{AB}}\left(P_{\mathrm{AB}}+P_{\mathrm{BC}}-1\right)}{P_{\mathrm{AB}}-1} \\
& \wedge P_{\mathrm{AB}}<1-P_{\mathrm{BC}} .
\end{aligned}
$$

If none of inequalities (5a)-(5c) are satisfied, as is the case in $75 \%$ of the winning probability parameter space, then $\mathbf{x}_{8}^{*} \in \mathbb{R}_{+}^{3}$, while $\mathbf{x}_{5}^{*}, \mathbf{x}_{6}^{*}$, and $\mathbf{x}_{7}^{*}$ have both an unstable and a stable eigendirection in the carrying simplex. The qualitative

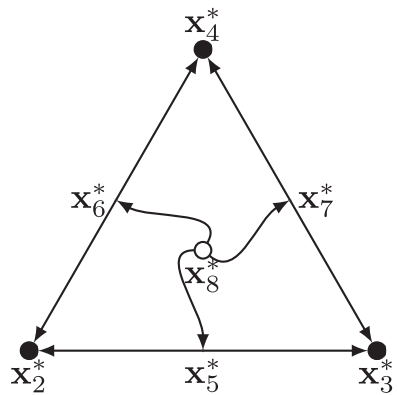

(a)

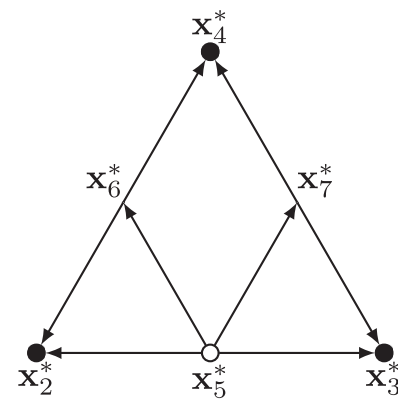

(b)
FIG. 2. Qualitative illustration of the dynamics of System (4) on the carrying simplex, if $\mathbf{x}_{8}^{*} \in \mathbb{R}_{+}^{3}$ (a) or $\mathbf{x}_{8}^{*} \notin \mathbb{R}_{+}^{3}$ (b). When a fixed point attracts on the simplex, it is displayed as (•), and, when it repels, as (०). Fixed points acting as saddle nodes on the simplex can be found at the intersection of their unstable and stable manifolds.

dynamics on the simplex in this case are displayed in Fig. 2(a), where $\mathbf{x}_{8}^{*}$ can be both an unstable spiral or an unstable node. When one or more of inequalities (5a)-(5c) are satisfied, it holds that $\mathbf{x}_{8}^{*} \notin \mathbb{R}_{+}^{3}$, while either $\mathbf{x}_{5}^{*}$, $\mathbf{x}_{6}^{*}$, or $\mathbf{x}_{7}^{*}$ has two unstable eigendirections in the carrying simplex, as displayed in Fig. 2(b).

In the case of non-transitive deterministic competition, thus either $P_{\mathrm{AB}}=P_{\mathrm{BC}}=P_{\mathrm{CA}}=1$ or $P_{\mathrm{AB}}=P_{\mathrm{BC}}=P_{\mathrm{CA}}=0$, one finds only four fixed points on the carrying simplex, namely, $\mathbf{x}_{2}^{*}, \mathbf{x}_{3}^{*}, \mathbf{x}_{4}^{*}$, and $\mathbf{x}_{8}^{*}$. Although the coexistence fixed point is unstable, the equations allow for coexistence of the three species due to a heteroclinic orbit connecting $\mathbf{x}_{2}^{*}, \mathbf{x}_{3}^{*}$, and $\mathbf{x}_{4}^{*}$. Trajectories approach this heteroclinic orbit asymptotically, and thus never reach it, such that species A, B, and $\mathrm{C}$ alternately dominate the ecosystem as time goes by Ref. 15 .

The analysis carried out in this section shows that the introduction of winning probabilities makes coexistence impossible when the system is well mixed and the system size $N \rightarrow+\infty$. Trajectories end up at either $\mathbf{x}_{2}^{*}, \mathbf{x}_{3}^{*}$, or $\mathbf{x}_{4}^{*}$, depending on the initial condition, so that only one species survives.

\section{B. Dynamics for finite $N$}

When $N$ is finite, coexistence suffers the same fate, as the IBM also evolves to one of the three states where only one species survives, even when non-transitive deterministic competition is considered. ${ }^{15}$ To illustrate how winning probabilities influence the duration of this transient to monoculture, the well-mixed IBM was evolved for $P_{\mathrm{AB}}, P_{\mathrm{BC}}$, and $P_{\mathrm{CA}}$ ranging from zero to one in increments of 0.05 , with $\mu=\sigma=1 / 2$ and $N=1000$, and the initial number of individuals $\mathrm{A}, \mathrm{B}$, and $\mathrm{C}$ present in the system equal to 300 per species. For each parameter combination, the number of generations until the first species extinction was calculated and averaged over 100 experiments, and the resulting average coexistence time is displayed in Fig. 3, for $P_{\mathrm{CA}}=1$. Although the figure hence only shows the results obtained for a two-dimensional slice through the three-dimensional parameter space considered, Fig. 3 is representative for what happens in the entire parameter space, as the results for $P_{\mathrm{CA}} \neq 1$ only quantitatively differ from the results obtained for $P_{\mathrm{CA}}=1$. Conclusions about the impact 


\section{$\langle t\rangle$ [generations]}

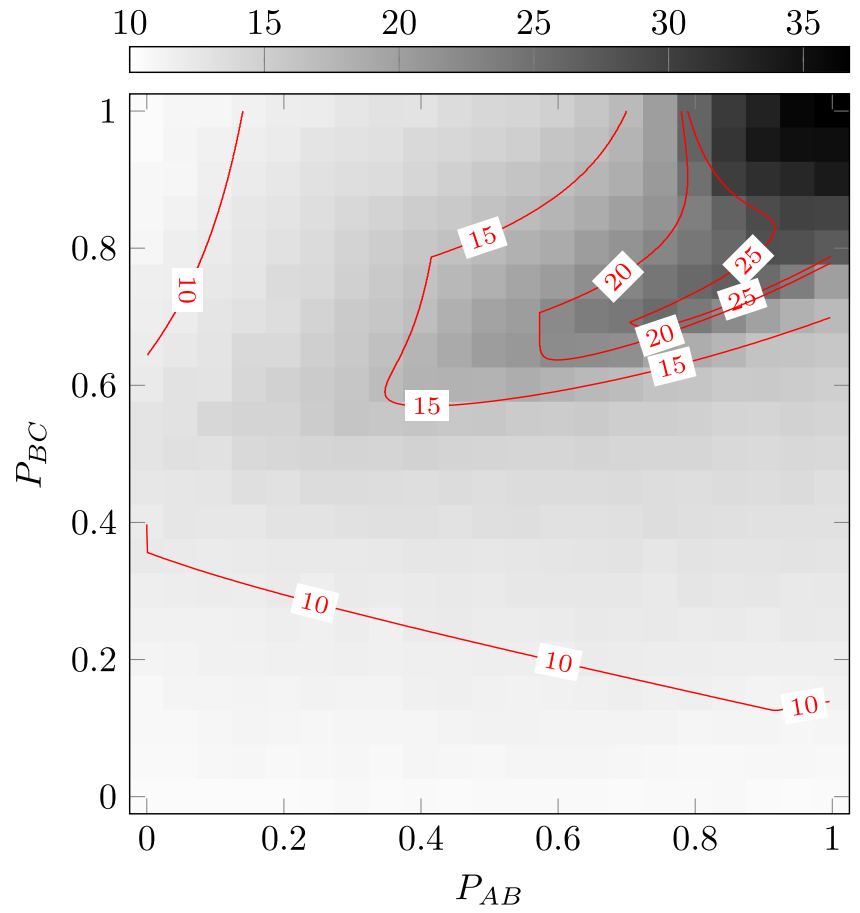

FIG. 3. Average coexistence times $\langle t\rangle$ for the well-mixed IBM, for $P_{\mathrm{AB}}$ and $P_{\mathrm{BC}}$ ranging from zero to one in increments of 0.05 , with $P_{\mathrm{CA}}=1$, $\mu=\sigma=1 / 2$, and $N=1000$. Initially, 300 individuals of species $\mathrm{A}, \mathrm{B}$, and $\mathrm{C}$ were present, and each experiment was repeated 100 times. For the same parameter settings, System (4) was solved, and the number of generations until the evolved trajectory arrives within a distance of $10^{-3}$ units of one of the coordinate axes $a, b$, or $c$ is displayed by red contours.

of the winning probabilities on the dynamics of the system drawn from Fig. 3 hence also remain valid when $P_{\mathrm{CA}} \neq 1$.

Figure 3 shows that, although one can state that, in general, deviations from non-transitive deterministic competition tend to shorten coexistence times, the effect is rather weak, as is to be expected for a system that rapidly collapses to monoculture regardless of its parameters. Coexistence times are larger when the competition network is not weakly stochastic transitive, i.e., when $P_{\mathrm{AB}}, P_{\mathrm{BC}} \geq 1 / 2$, but it is not possible to make strong statements about expected coexistence times based on the property of weakly stochastic transitivity.

To illustrate the connection between the dynamics of System (4) and the IBM, System (4) was solved for $P_{\mathrm{AB}}$ and $P_{\mathrm{BC}}$ ranging from zero to one, $P_{\mathrm{CA}}=1, \mu=\sigma=1 / 2$, and $a(0)=$ $b(0)=c(0)=3 / 10$. The number of generations required for the trajectories to arrive within a distance of $10^{-3}$ units of one of the coordinate axes $a, b$, or $c$ is displayed in Fig. 3 using red contours. This quantity scales well with the coexistence times retrieved with the IBM.

\section{Survival of the strongest}

Due to the finite size of the IBM, each experiment will eventually result in the survival of a single species. The survival frequency for each species, computed on the basis of 100 IBM experiments for each combination of $P_{\mathrm{AB}}$ and $P_{\mathrm{BC}}$ while $P_{\mathrm{CA}}=1$, is displayed in Fig. 4. As species A is always outcompeted by species $\mathrm{C}\left(P_{\mathrm{CA}}=1\right)$, while itself outcompeting

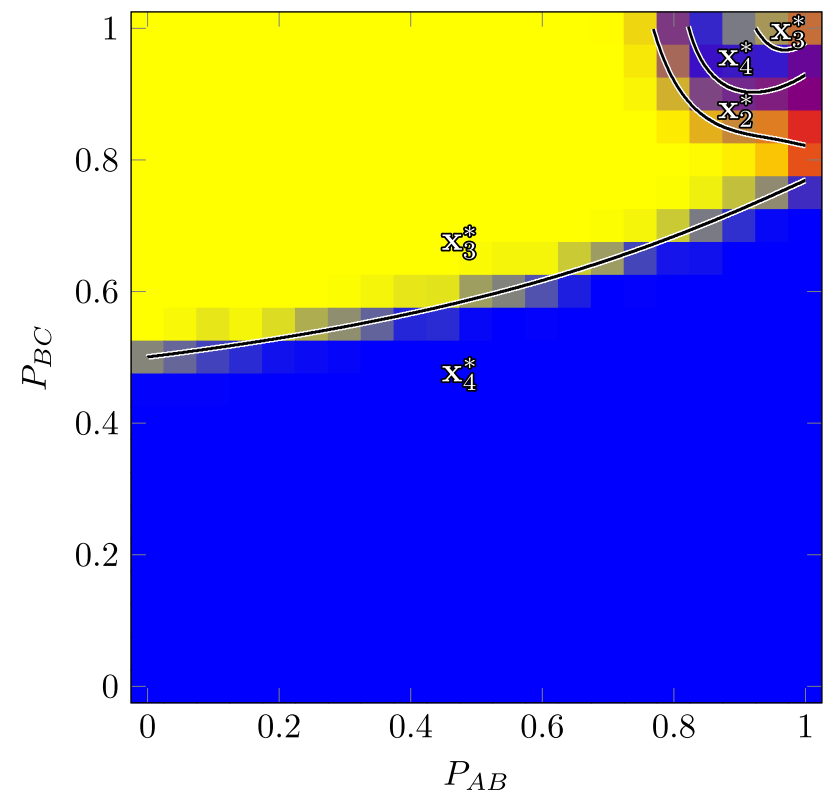

FIG. 4. Competition outcomes for the well-mixed IBM, for $P_{\mathrm{AB}}$ and $P_{\mathrm{BC}}$ ranging from zero to one in increments of 0.05 , with $P_{\mathrm{CA}}=1, \mu=\sigma=1 / 2$, and $N=1000$. Initially, 300 individuals of species $\mathrm{A}, \mathrm{B}$, and $\mathrm{C}$ were present, and each experiment was repeated 100 times. The sole surviving species for each IBM simulation is displayed by blending the colours red for species A, yellow for species $\mathrm{B}$, and blue for species $\mathrm{C}$ according to their survival frequency among 100 experiments. For the same parameter settings, the parameter space is subdivided in areas where the system ends up in either $\mathbf{x}_{2}^{*}, \mathbf{x}_{3}^{*}$, or $\mathbf{x}_{4}^{*}$ according to System (4).

species B with winning probability $P_{\mathrm{AB}}$, species $\mathrm{A}$ has difficulty persisting for $P_{\mathrm{AB}}$ significantly smaller than $P_{\mathrm{CA}}=1$. As such, for most of the parameter settings considered, species $\mathrm{B}$ and $\mathrm{C}$ are the only candidates for survival. Winning probability $P_{\mathrm{BC}}$ determines which of those will prevail, where species C's chances increase with increasing $P_{\mathrm{AB}}$, as species A can then help C with killing B. Only for $P_{\mathrm{AB}}, P_{\mathrm{BC}} \gtrsim 0.8$, when the winning probabilities are close to those of the non-transitive deterministic competition network, does the outcome of the experiments become less predictable. Here, the intricate dynamics of the IBM, combined with stochastic effects due to its finite size, make that each species has an equitable survival probability. We can discern an approximate "survival of the strongest" law, where the species with the highest probability of winning competition events involving the other two species has the highest probability for survival, although this statement becomes less accurate when the winning probabilities approach the non-transitive deterministic setting. The "survival of the weakest" law, as proposed in Ref. 30, however, would seem to contradict this finding. Yet, in Ref. 30, instead of the May-Leonard model, the Lotka-Volterra model is used. This model consists out of exchange events, as given by Reaction (3), and events that combine competition and reproduction, given by

$$
\begin{aligned}
& \mathrm{AB} \stackrel{k_{\mathrm{A}}}{\rightarrow} \mathrm{AA}, \\
& \mathrm{BC} \stackrel{k_{\mathrm{B}}}{\rightarrow} \mathrm{BB}, \\
& \mathrm{CA} \stackrel{k_{\mathrm{C}}}{\rightarrow} \mathrm{CC},
\end{aligned}
$$


which imply three essential differences between the Lotka-Volterra model and the May-Leonard model studied in this paper. First, in the Lotka-Volterra model, the number of individuals is conserved, as competition results in the replacement of an individual by one from another species. Second, the competition is deterministic, as A always beats B, while B never beats $\mathrm{A}$. Third, in the May-Leonard model, competition events between species happen with equal reaction rate constants, $P_{\mathrm{AB}} \sigma+\left(1-P_{\mathrm{AB}}\right) \sigma=P_{\mathrm{BC}} \sigma+$ $\left(1-P_{\mathrm{BC}}\right) \sigma=P_{\mathrm{CA}} \sigma+\left(1-P_{\mathrm{CA}}\right) \sigma=\sigma$, while in the LotkaVolterra model, it may be that $k_{\mathrm{A}} \neq k_{\mathrm{B}} \neq k_{\mathrm{C}}$.

It was found that if, for instance, $k_{\mathrm{A}}<\min \left(k_{\mathrm{B}}, k_{\mathrm{C}}\right)$, species A has the best chances of becoming the sole survivor, ${ }^{30}$ a result that was coined "survival of the weakest," as it seems to indicate that the species that has the lowest reaction rate constant associated with attacking and killing its competitor, is the most probable survivor. This result can be understood by noticing that when $k_{\mathrm{A}}$ is low, species $\mathrm{B}$ is rarely attacked and hence maintains a large population, which in turn attacks species $\mathrm{C}$ in large numbers, hence reducing the population size of species A's attacker. It is clear that this train of thought depends crucially on the lack of aggressiveness, i.e., eagerness to engage in a competition event, encoded in $k_{\mathrm{A}}$. As one could understand strength (or weakness) to mean the probability of winning (or losing) a head-to-head encounter with a competitor, the phrase "survival of the weakest" may be a bit confusing, and may more accurately be renamed "survival of the least aggressive."

Indeed, our results show that when all species are equally aggressive, i.e., equally likely to engage in a competition event (expressed by $P_{\mathrm{AB}} \sigma+\left(1-P_{\mathrm{AB}}\right) \sigma=P_{\mathrm{BC}} \sigma+$ $\left.\left(1-P_{\mathrm{BC}}\right) \sigma=P_{\mathrm{CA}} \sigma+\left(1-P_{\mathrm{CA}}\right) \sigma=\sigma\right)$, then the strongest species, being the one that has the highest probability of winning a head-to-head encounter with a competitor, often becomes the sole survivor, as such reinstating the intuitive "survival of the strongest" expression.

For $P_{\mathrm{AB}}$ and $P_{\mathrm{BC}}$ ranging from zero to one, $P_{\mathrm{CA}}=1$, $\mu=\sigma=1 / 2$, and $a(0)=b(0)=c(0)=3 / 10$, it was determined which of the fixed points of System (4) attracts the solution. In Fig. 4, the black curves subdivide the parameter space into areas where the system ends up in either $\mathbf{x}_{2}^{*}, \mathbf{x}_{3}^{*}$, or $\mathbf{x}_{4}^{*}$. One can conclude that System (4) approximates the outcomes of the IBM experiments well, except for $P_{\mathrm{AB}}, P_{\mathrm{BC}} \gtrsim$ 0.8 . Under these circumstances, the dynamics become intricate, while finite size effects become important, hence making the outcome of the IBM experiments sensitive to fluctuations.

\section{THE ONE-DIMENSIONAL SYSTEM}

We now turn our attention to the dynamics of the IBM in one space dimension. Although one may expect that, due to the finite size of the system, coexistence will be a transient feature in this setting, the consideration of space and the localization of the interactions may slow down the evolution toward monoculture due to the formation of spatial patterns..$^{35,52,59}$ We can therefore anticipate that the winning probabilities will influence the length of this transient by modifying the emerging patterns.

\section{A. Tunnelling events}

Figure 1(a) displays an example of an evolution starting from a random initial condition, as produced by the algorithm described in Sec. II. First, coarsening dynamics rapidly segregate the system into three domains, each dominated by a single species. ${ }^{21,22}$ The boundaries of these domains approximately perform a biased random walk, with an average speed proportional to the winning probabilities deciding the outcome of competition events among individuals occupying neighbouring domains. This can be understood by realising that the probability of a domain occupied by species A expanding by one cell at the cost of its neighbouring domain occupied by species B shrinking by one cell, is proportional to the probability of A killing its neighbour B at the boundary $\left(\sim P_{\mathrm{AB}}\right)$, and subsequently reproducing in the resulting empty cell.

Due to the finite size of the system, one of the domains must eventually give way to the other two. Since it holds that $P_{\mathrm{AB}}-P_{\mathrm{CA}}<P_{\mathrm{BC}}-P_{\mathrm{AB}}<P_{\mathrm{CA}}-P_{\mathrm{BC}}$ for the system displayed in Fig. 5(a), we may expect that the domain occupied by species A will disappear first, as species $\mathrm{C}$ invades it at a speed proportional to $P_{\mathrm{CA}}=1$, while the domain occupied

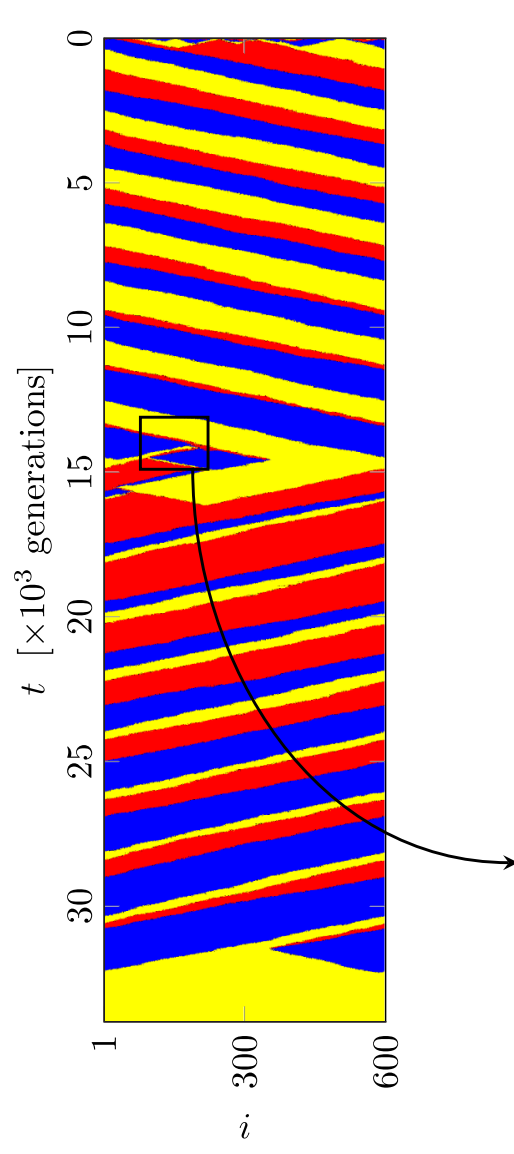

(a)

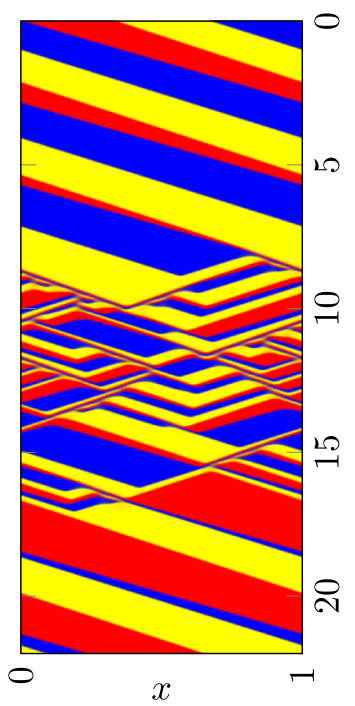

(c)

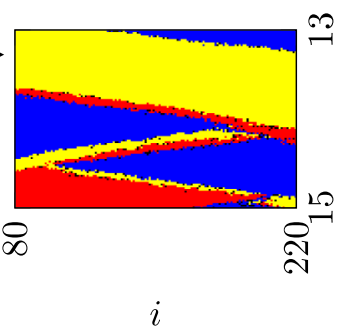

(b)
FIG. 5. Spatiotemporal evolution of the one-dimensional IBM, starting from a random initial condition, with $P_{\mathrm{AB}}=0.95, P_{\mathrm{BC}}=0.97, P_{\mathrm{CA}}=1, \mu=\sigma=$ $1 / 5, \epsilon=3 / 5$, and $N=600$, for $34 \times 10^{3}$ generations. Cells occupied by species A, B, or C are coloured red, yellow, or blue, respectively, while empty cells are black (a). A magnification for $(N, t) \in[80,220] \times\left[13 \times 10^{3}, 15 \times\right.$ $\left.10^{3}\right]$ (b). The solution of System (6) for the same parameter settings, and $(x, t) \in[0,1] \times\left[0,22 \times 10^{3}\right](\mathrm{c})$. 


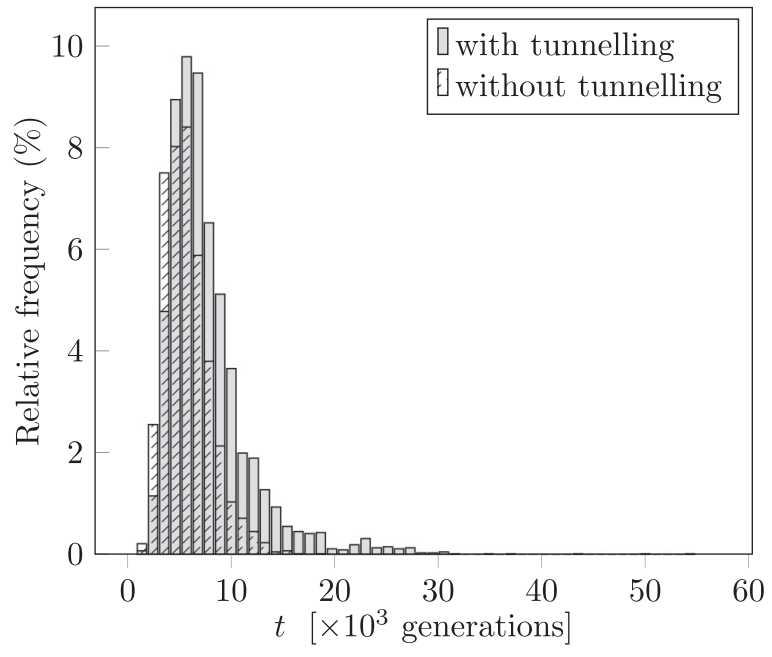

FIG. 6. Relative frequency of coexistence times in the one-dimensional IBM, with $P_{\mathrm{AB}}=0.95, P_{\mathrm{BC}}=0.97, P_{\mathrm{CA}}=1, \mu=\sigma=1 / 5, \epsilon=3 / 5$, and $N=$ 600. Initially, the system consisted of three equally sized domains, occupied by species A, B, and C, respectively. The experiment was repeated 5000 times.

by species A expands by displacing $\mathrm{B}$ at a speed only proportional to $P_{\mathrm{AB}}=0.95$. Often, this disappearance of the domain results in extinction of species A. However, for $\epsilon>0$, chance can revive the system in the sense that, as displayed in Fig. 5(c), an individual of species B can diffuse through the narrow domain occupied by species A, without being killed. After such a tunnelling event, ${ }^{23}$ the individual reaches the domain occupied by species $\mathrm{C}$, where it competes and reproduces, creating a new domain, and hence initiating a new round of biased random walks. After about $32 \times 10^{3}$ generations, this new domain occupied by species $\mathrm{A}$ is consumed by the other domains once again. This time, again by chance, there are no tunnelling events, and coexistence is lost.

To quantify to what extent tunnelling events can prolong coexistence after a first round of random walks, the one-dimensional IBM was evolved 5000 times, with $P_{\mathrm{AB}}=$ $0.95, P_{\mathrm{BC}}=0.97, P_{\mathrm{CA}}=1, \mu=\sigma=1 / 5, \epsilon=3 / 5$, and $N=$ 600 . Initially, the system was divided into three contiguous domains, each consisting of 200 cells, and occupied by species A, B, and C, respectively. In about $59 \%$ of the experiments, tunnelling events occurred. Figure 6 displays two histograms of the time until the first extinction, one for experiments in which no tunnelling events occurred, and one for those in which they did occur. The histograms demonstrate the positive effect that tunnelling events have on coexistence time. While tunnelling events shift the histogram to longer coexistence times, they also lead to a more pronounced tail, where IBM evolutions such as the one displayed in Fig. 5(a) can be retrieved. On average, coexistence persists for about 6505 generations without tunnelling events, compared to 8563 generations with tunnelling events, a significant prolongation (Mann-Whitney $U$ test, $p=2.2 \times 10^{-16}$ ). Other studies indicate that tunnelling events can prolong coexistence even more when considering high values for $\epsilon,{ }^{24}$ a quasi-one-dimensional space, ${ }^{25}$ or when each cell may be occupied by more than one individual. ${ }^{23}$ However, to the best of our knowledge, such patterns have not yet been observed in real-life experiments, so it remains uncertain whether such tunnelling events actually occur.

\section{B. The continuum limit}

Upon taking the continuum limit $(N \rightarrow \infty)$ and neglecting spatial correlations, the dynamics of the IBM can be approximated by a system of partial differential equations (PDEs): ${ }^{18}$

$$
\left\{\begin{array}{l}
\dot{a}=\frac{\epsilon}{\mu d N^{2 / d}} \Delta a+f_{1}, \\
\dot{b}=\frac{\epsilon}{\mu d N^{2 / d}} \Delta b+f_{2}, \\
\dot{c}=\frac{\epsilon}{\mu d N^{2 / d}} \Delta c+f_{3},
\end{array}\right.
$$

where $d$ is the spatial dimension of the system, $\Delta$ is the Laplacian, and $f_{1}, f_{2}$, and $f_{3}$ are as defined in System (4).

Solutions of System (6) produce a rich variety of spatial patterns, due to the ceaseless occurrence of tunnelling events. Depending on the winning probabilities, the system can produce anything ranging from regular Sierpinskitriangle-like patterns to irregular spatiotemporal chaos. As an illustration, Fig. 5(b) displays a numerical solution of System (6), obtained using the Exponential Time Differencing fourth-order Runge-Kutta method, ${ }^{60}$ for $(x, t) \in[0,1] \times$ $\left[0,22 \times 10^{3}\right]$, with $P_{\mathrm{AB}}=0.95, P_{\mathrm{BC}}=0.97, P_{\mathrm{CA}}=1, \mu=$ $\sigma=1 / 5, \epsilon=3 / 5$, and $N=600$. For these settings, System (6) is able to maintain coexistence for $t \rightarrow+\infty$, in contrast to the corresponding IBM evolved for the same parameter settings [Fig. 5(a)]. Due to the stochastic nature of the IBM, tunnelling events happen only with a certain probability, while they are guaranteed to occur according to the corresponding PDEs. Consequently, as coexistence times in the one-dimensional IBM depend critically on tunnelling events, and hence on stochasticity, System (6) is not as good an approximation for the dynamics of the one-dimensional IBM, as System (4) is for the well-mixed IBM.

\section{Coexistence time}

The one-dimensional IBM was evolved 50 times for $P_{\mathrm{AB}}$, $P_{\mathrm{BC}}$, and $P_{\mathrm{CA}}$ ranging from zero to one in increments of 0.05 , with $\mu=\sigma=1 / 5, \epsilon=3 / 5$, and $N=600$. Initially, the system was again divided into three equal-sized contiguous domains, each occupied by a single species. The average coexistence times are displayed in Fig. 7(a), for $P_{\mathrm{CA}}=1$. As the boundaries of each domain approximately perform a biased random walk with an average speed proportional to the winning probabilities, coexistence times are the largest for the non-transitive deterministic competition network, where $P_{\mathrm{AB}}=P_{\mathrm{BC}}=P_{\mathrm{CA}}=1$, since all boundaries then move at an equal average speed. Deviations from this non-transitive deterministic setting result in strongly reduced coexistence times, as the boundaries are then biased to walk into each other. Again, in general, one can expect coexistence times to be larger when the competition network is not weakly stochastic transitive, i.e., when $P_{\mathrm{AB}}, P_{\mathrm{BC}} \geq 1 / 2$, but it is not possible to make strong statements about expected coexistence times based on the property of weakly stochastic transitivity. 


\section{$\langle t\rangle$ [generations]}

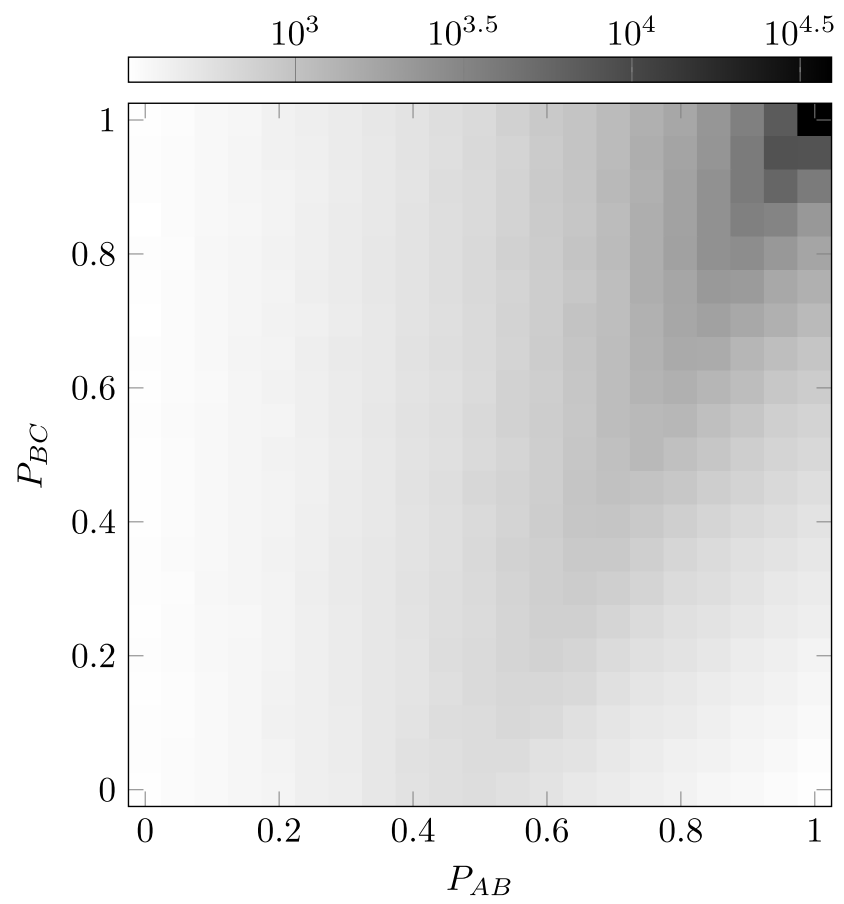

(a)

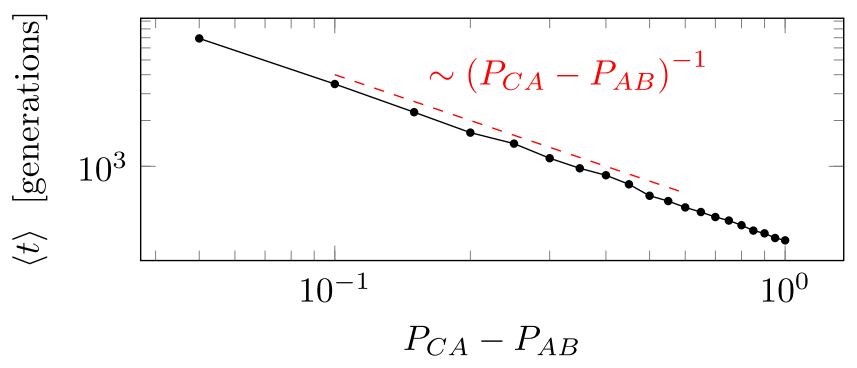

(b)

FIG. 7. Average coexistence time $\langle t\rangle$ in the one-dimensional IBM for $P_{\mathrm{AB}}$ and $P_{\mathrm{BC}}$ ranging from zero to one in increments of 0.05 , with $P_{\mathrm{CA}}=1, \mu=$ $\sigma=1 / 5, \epsilon=3 / 5$, and $N=600$. Initially, the system was divided into three equal-sized domains, each occupied by a single species. Each experiment was repeated 50 times (a). For $P_{\mathrm{BC}}=P_{\mathrm{CA}}=1$, the average coexistence time is related to $P_{\mathrm{CA}}-P_{\mathrm{AB}}$ by a power law (b).

This sensitive dependence of the coexistence times on the winning probabilities is underlined by Fig. 7(b), where the coexistence times displayed in Fig. 7(a) for $P_{\mathrm{BC}}=P_{\mathrm{CA}}=1$ and $0 \leq P_{\mathrm{AB}}<1$ are plotted versus $P_{\mathrm{CA}}-P_{\mathrm{AB}}=1-P_{\mathrm{AB}}$. Since for these parameter settings, it holds that $P_{\mathrm{AB}}-P_{\mathrm{CA}}=$ $P_{\mathrm{AB}}-1<P_{\mathrm{CA}}-P_{\mathrm{BC}}=0<P_{\mathrm{BC}}-P_{\mathrm{AB}}=1-P_{\mathrm{AB}}$, the domain occupied by species $A$ is expected to shrink the fastest, at a speed proportional to $P_{\mathrm{CA}}-P_{\mathrm{AB}}$. As tunnelling events have a rather modest impact on the coexistence times under the considered parameter settings, one may expect that the extinction times are inversely proportional to the time it takes for one domain to be destroyed, and hence to $P_{\mathrm{CA}}-$ $P_{\mathrm{AB}}$. As shown in Fig. 7(b), one indeed finds that for the IBM, $\langle t\rangle \sim\left(P_{\mathrm{CA}}-P_{\mathrm{AB}}\right)^{-1}$, illustrating how the introduction of the winning probabilities can cut coexistence short.

The competition outcomes of the experiments described in the previous paragraphs are displayed in Fig. 8. Above the

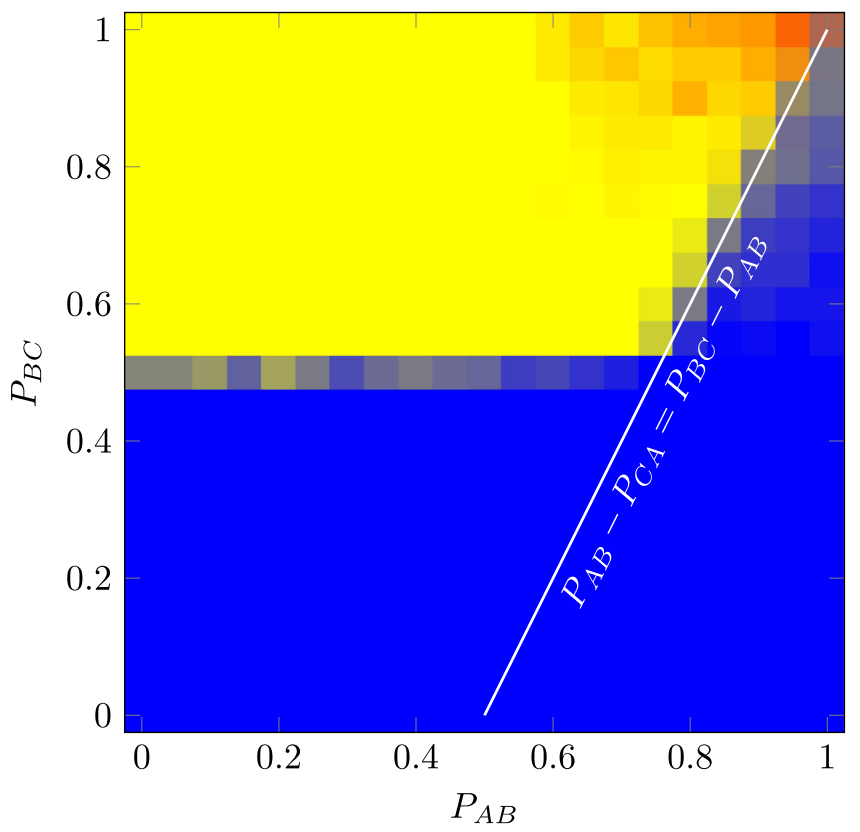

FIG. 8. Competition outcomes in the one-dimensional IBM for $P_{\mathrm{AB}}$ and $P_{\mathrm{BC}}$ ranging from zero to one in increments of 0.05 , with $P_{\mathrm{CA}}=1, \mu=\sigma=1 / 5$, $\epsilon=3 / 5$, and $N=600$. Initially, the system was divided in three equalsized domains, each occupied by a single species, and each experiment was repeated 50 times. The sole surviving species for each IBM simulation is displayed by blending red for species A, yellow for species B, and blue for species $\mathrm{C}$ according to their survival frequency among the 50 experiments.

white line, it holds that $P_{\mathrm{AB}}-P_{\mathrm{CA}}=P_{\mathrm{AB}}-1<P_{\mathrm{BC}}-P_{\mathrm{AB}}$ and $P_{\mathrm{AB}}-P_{\mathrm{CA}}=P_{\mathrm{AB}}-1<P_{\mathrm{CA}}-P_{\mathrm{BC}}=1-P_{\mathrm{BC}}$, such that, in general, the domain occupied by species $\mathrm{A}$ disappears first. If this disappearance is not followed by tunnelling events, whether species $\mathrm{B}$ or $\mathrm{C}$ becomes the sole survivor depends on $P_{\mathrm{BC}}$. Below the white line, it holds that $P_{\mathrm{AB}}-$ $P_{\mathrm{CA}}=P_{\mathrm{AB}}-1>P_{\mathrm{BC}}-P_{\mathrm{AB}}$ and $P_{\mathrm{CA}}-P_{\mathrm{BC}}=1-P_{\mathrm{BC}}>$ $P_{\mathrm{BC}}-P_{\mathrm{AB}}$, hence the domain occupied by species $\mathrm{B}$ generally disappears first, after which species $\mathrm{C}$ becomes the sole survivor, since competition events between species $\mathrm{A}$ and $\mathrm{C}$ are decided by $P_{\mathrm{CA}}=1$. For $P_{\mathrm{AB}} \gtrsim 0.6$ and $P_{\mathrm{BC}} \gtrsim 0.8$, tunnelling events offer species $A$ a small chance to become the sole survivor, although at first sight such winning probabilities do not seem to favor it. We conclude that, in general, the species that has the highest probability of winning competition events against the other two species, has the best chances for survival, a result that again agrees with the "survival of the strongest" law.

\section{THE TWO-DIMENSIONAL SYSTEM}

In the two-dimensional IBM, when the non-transitive deterministic competition network is considered, coexistence is known to be long-lived due to the formation of spiral waves ${ }^{19}$ [Fig. 9(b)]. As displayed in Fig. 10, slight deviations from this non-transitive deterministic setting do not seem to profoundly impact coexistence, whereas larger deviations decrease the coexistence time by several orders of magnitude. This observation is confirmed by Fig. 9(a), which shows that, when $P_{\mathrm{CA}}=1$, coexistence times start to increase strongly for $P_{\mathrm{AB}} \gtrsim 0.6$ up to $P_{\mathrm{AB}} \approx 0.9$, where a plateau is reached. 


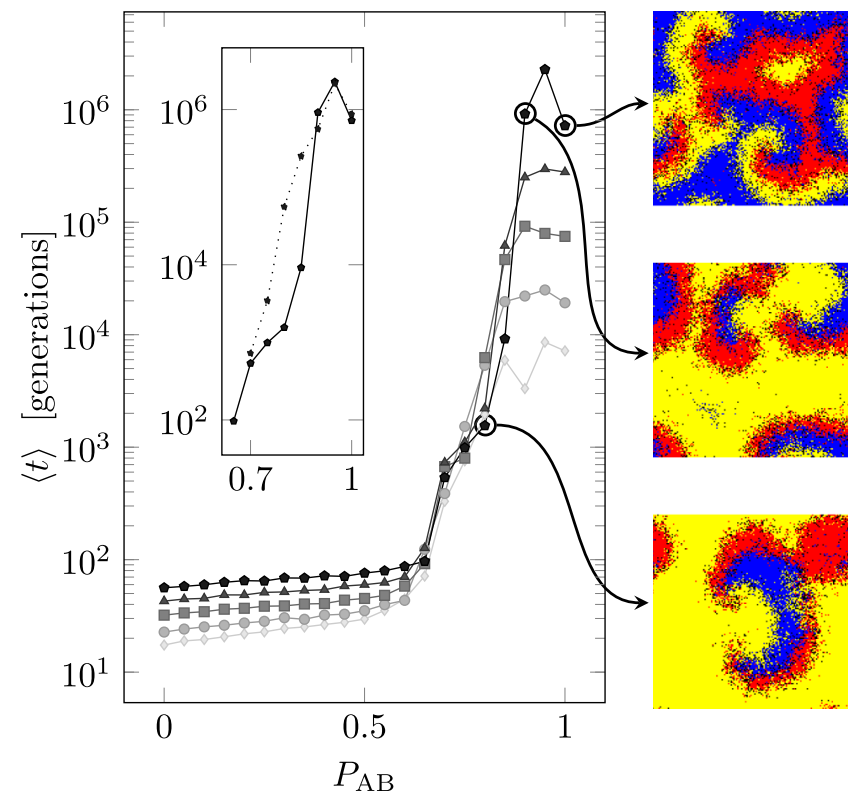

(a) (b)
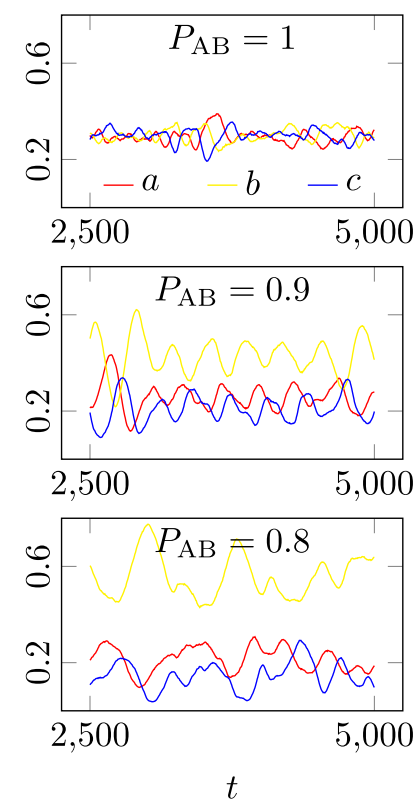

(c)
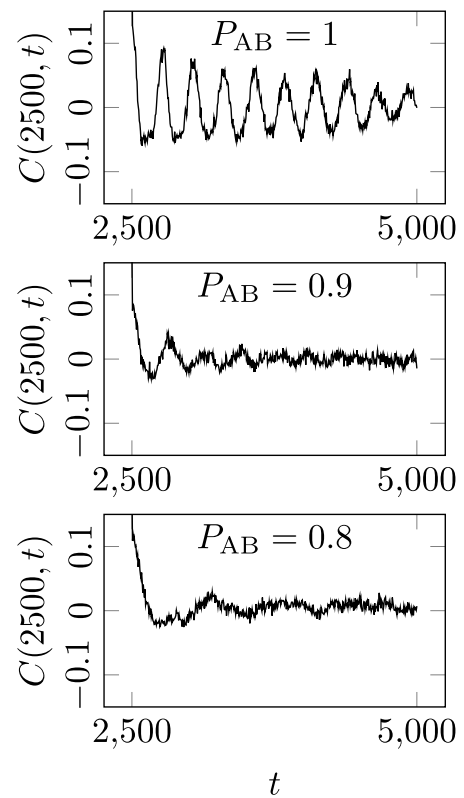

(d)

FIG. 9. Average coexistence time $\langle t\rangle$ in the two-dimensional IBM for $P_{\mathrm{AB}}$ ranging from zero to one in increments of 0.05 , with $P_{\mathrm{BC}}=P_{\mathrm{CA}}=1, \mu=\sigma=1 / 5$, $N=50(\neg), N=75(-\circ), N=100(-\square), N=125\left(-\triangle_{-}\right)$, or $N=150(\rightarrow-)$, and scaling $\epsilon$ such that the diffusion coefficient $\epsilon / \mu d N$ is the same for all experiments and grid sizes. Each experiment was repeated 20 times, and the initial conditions were random, as discussed in Sec. II. The inset is a reproduction of the curve for $N=150(-)$, combined with the coexistence times for the same experiments, but with an initial condition involving spiral waves (....) (a). Examples of spatial configurations, where cells occupied by species A are coloured red, by species B are coloured yellow, and by species $\mathrm{C}$ are coloured blue, while empty cells are coloured black (b). The species densities $a, b$, and $c$ for examples of IBM evolutions, where $P_{\mathrm{AB}}=1$ (upper panel), $P_{\mathrm{AB}}=0.9$ (middle panel), and $P_{\mathrm{AB}}=0.8$ (lower panel) (c). The two-time autocorrelation function $C\left(t_{0}, t_{1}\right)=\left\langle\delta_{s \mathrm{~A}}\left(t_{0}\right) \delta_{s \mathrm{~A}}\left(t_{1}\right)\right\rangle-\left\langle\delta_{s \mathrm{~A}}\left(t_{0}\right)\right\rangle\left\langle\delta_{s \mathrm{~A}}\left(t_{1}\right)\right\rangle$, as derived from 500 IBM simulations, starting from identical initial conditions (d).

Again, in general, coexistence times are larger when the competition network is not weakly stochastic transitive, i.e., when $P_{\mathrm{AB}}, P_{\mathrm{BC}} \geq 1 / 2$, but these competition networks can also produce short coexistence times.

As displayed in Fig. 9(b), the system can still produce spiral wave patterns even when the competition network is not deterministic, a result that complements the findings in Ref. 26, where slightly asymmetric reproduction rate constants were considered. Although species evenness, a quantity that measures how far relative species abundances are from being equal, can be reduced due to the winning probabilities, as suggested in Fig. 9(b) and confirmed by Fig. 9(c), this does not necessarily impact the coexistence time: Fig. 9(b) shows that, although species B is the most abundant, implying low species evenness, spiral waves are still being formed, which leads to long coexistence times. This result underlines that spatial segregation, rather than species evenness, is the main determinant of coexistence.

To study the spiral wave dynamics for varying winning probabilities, we introduce the two-time autocorrelation function $C\left(t_{0}, t_{1}\right)=\left\langle\delta_{s \mathrm{~A}}\left(t_{0}\right) \delta_{s \mathrm{~A}}\left(t_{1}\right)\right\rangle-\left\langle\delta_{s \mathrm{~A}}\left(t_{0}\right)\right\rangle\left\langle\delta_{s \mathrm{~A}}\left(t_{1}\right)\right\rangle$, where $\langle\ldots\rangle$ denotes the average over 500 IBM simulations, and $s$ denotes the state of the cell in the upper-left corner of the grid, such that the Kronecker delta $\delta_{s \mathrm{~A}}(t)$ becomes one when this cell is occupied by species $\mathrm{A}$ at time $t$, and zero otherwise. Interestingly, two-time autocorrelations are less pronounced in the non-deterministic setting [Fig. 9(d)], due to the spiral waves breaking up. When $P_{\mathrm{AB}}=P_{\mathrm{BC}}=P_{\mathrm{CA}}=1$, the non-transitive deterministic setting, the system tends to lock into a repeating spiral wave pattern, only slightly altered by the stochastic nature of the system and the drift of the spiral wave cores. This periodic behavior of the spatiotemporal patterns is reflected in the oscillatory auto-correlation function displayed in the upper panel of Fig. 9(d). When $P_{\mathrm{BC}}=P_{\mathrm{CA}}=1$, and $P_{\mathrm{AB}}=0.9$ or $P_{\mathrm{AB}}=0.8$, spiral waves are short-lived. Soon after their formation, they break up and are replaced by new spiral waves with the same frequency and wavelength, but a different spatial configuration. The corresponding two-time autocorrelation hence quickly dampens, although, for instance, for $P_{\mathrm{AB}}=0.9$, the continuous destruction and production of spiral waves can still result in long-lived coexistence.

When $P_{\mathrm{BC}}=P_{\mathrm{CA}}=1$ and $P_{\mathrm{AB}} \gtrsim 0.9$, extinction times do not depend on the winning probabilities, since under such parameter settings the formation of spatial patterns guarantees coexistence, and thus extinction events are the result of rare fluctuations. Hence, the coexistence time is a function of the system size, $N^{2}$, as this quantity determines the frequency of these fatal fluctuations.

As illustrated in the inset of Fig. 9(a), when $P_{\mathrm{BC}}=P_{\mathrm{CA}}=$ 1 and $0.9 \gtrsim P_{\mathrm{AB}} \gtrsim 0.6$, the initial conditions are important. When the IBM evolves from spatially structured initial conditions, as obtained by a continuation of the spatial configurations evolved under slightly different parameter settings, average coexistence times are orders of magnitude longer than when starting from random initial conditions. In the latter case, spiral wave patterns do not develop easily, and when they fail to do so, coexistence is rapidly lost. When $P_{\mathrm{AB}} \gtrsim 0.9$, however, most random initial conditions give rise to spatial structures. These findings are in contrast to those in one 
$\langle t\rangle$ [generations]

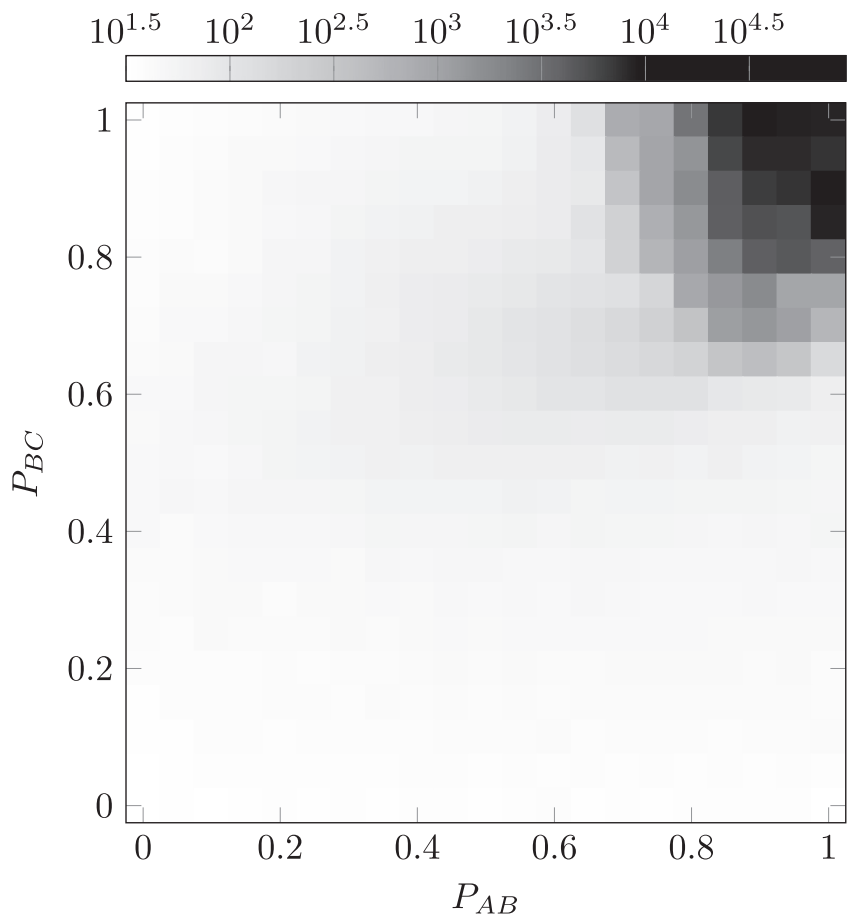

FIG. 10. Average coexistence time $\langle t\rangle$ in the two-dimensional IBM for $P_{\mathrm{AB}}$ and $P_{\mathrm{BC}}$ ranging from zero to one in increments of 0.05 , with $P_{\mathrm{CA}}=1, \mu=$ $\sigma=1 / 5, \epsilon=3 / 5$, and $N=100^{2}$. Each experiment was repeated 20 times.

dimension (Sec. IV), where a coarsening dynamics implies spatial structure in the form of domains, irrespective of the initial conditions.

We may conclude that in two dimensions, more so than in one dimension and in the well-mixed case, coexistence

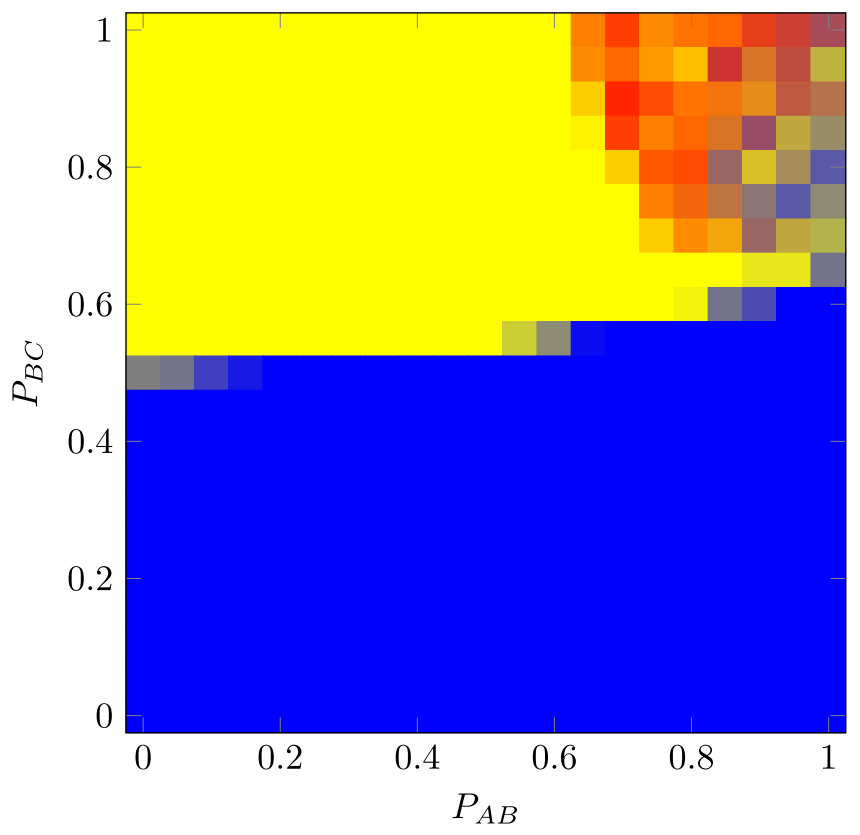

FIG. 11. Competition outcomes in the two-dimensional IBM for $P_{\mathrm{AB}}$ and $P_{\mathrm{BC}}$ ranging from zero to one in increments of 0.05 , with $P_{\mathrm{CA}}=1, \mu=\sigma=1 / 5$, $\epsilon=3 / 5$, and $N=100^{2}$. The sole surviving species for each IBM simulation is displayed by blending red for species $\mathrm{A}$, yellow for species $\mathrm{B}$, and blue for species $\mathrm{C}$ according to their survival frequency among 20 experiments. seems to be robust, in the sense that small deviations from the non-transitive deterministic competition network have little impact on the coexistence time. However, larger deviations cut coexistence short.

The competition outcomes of the two-dimensional IBM experiments described in the previous paragraphs are displayed in Fig. 11. We can infer that competition outcomes for $P_{\mathrm{CA}}=1$ and $P_{\mathrm{AB}}, P_{\mathrm{BC}} \gtrsim 0.6$ are uncertain. This is not due to sensitive dependence on initial conditions, but to the intricate spiral wave dynamics and the rare fluctuations that lead to extinctions, phenomena that also produce long coexistence times for these parameter ranges, as displayed in Fig. 10. When $P_{\mathrm{CA}}=1$ and $P_{\mathrm{AB}}, P_{\mathrm{BC}} \lesssim 0.6$, species $\mathrm{A}$ goes extinct first, after which $P_{\mathrm{BC}}$ and the species abundances when A goes extinct decide whether species $\mathrm{B}$ or $\mathrm{C}$ becomes the sole survivor. We can thus again discern the "survival of the strongest" law, which is obscured when the winning probabilities approach the non-transitive deterministic setting.

\section{DISCUSSION AND CONCLUSIONS}

Of the research on the biodiversity enigma, one strand has concentrated on ecological interaction networks and their intricate structure, often studying the role that competition plays in determining the presence, absence, and abundances of species in these networks. As understanding the interaction of two species requires simpler experiments and theory than those needed to address the dynamics for many species, ${ }^{61}$ much of the competition research has focused on pairwise mechanisms, although some phenomena which are suspected as being key to maintaining coexistence in natural species-rich ecosystems only emerge in networks consisting of three or more competitors. ${ }^{62}$ An example is non-transitive competition, of which theoretical research ${ }^{11-34}$ has shown that it is able to maintain the coexistence of three or more species, while each pair cannot coexist in isolation. However, empirical support for this mechanism of coexistence is not extensive, ${ }^{63}$ and hence ecologists lack a clear understanding of the prevalence and significance of non-transitive competition in ecosystems in nature.

The lack of empirical evidence concerning coexistence mechanisms in ecological interaction networks is unsurprising, as meaningful experiments require the quantification of the competitive ability of all species involved, and the inference of the full set of interaction coefficients in the network, two important barriers to progress on this front. Although ecologists have nonetheless devoted much time and effort to measuring interactions between species in some natural ecosystems, involving grasslands, ${ }^{64}$ insects, ${ }^{65,66}$ shellfish, ${ }^{67}$ and microbial communities, ${ }^{68,69}$ the lack of an established methodology for measuring competitive interactions implies that a plethora of dissimilar experimental approaches has led to contradicting conclusions.

Modelling can help to bridge the gap between current empirical techniques and the goal of prediction of longterm competitive outcomes, identifying which experimental degrees of freedom are expected to have an important impact on experimental results. For example, theoretical models 
have been used to investigate if and how competitive outcomes are affected by dispersal, ${ }^{19}$ network structure, ${ }^{17}$ spatial heterogeneity, ${ }^{35}$ the presence of more than three species, ${ }^{70,71}$ and many other important factors. However, one simplifying assumption that may restrict the real-life applicability of these theoretical results is that, in the non-transitive deterministic competition network, the outcomes of competition events are modelled as certain and predetermined, which makes the species identical up to their competitive relationships, and introduces an unnatural symmetry in the competition network. When trying to understand if and how non-transitive competition may impact biodiversity in real-life ecosystems, it is important to check whether coexistence times are still prolonged when this unnatural assumption regarding the symmetry of the competitive relations is relaxed.

We have addressed this issue in this work, where we have made the outcomes of competition events non-deterministic by introducing winning probabilities, and subsequently investigated whether species manage to coexist when the outcomes of competition events are uncertain.

We find that the results are dependent on the spatial dimension of the system. In a well-mixed non-deterministic setting, species do not coexist, which is unsurprising since there was already no coexistence in the non-transitive deterministic setting. In a one-dimensional non-deterministic system, coexistence times decay as a power law as the winning probabilities shift the system away from the non-transitive deterministic setting. Chaotic patterns prolonging coexistence emerge, but whether these occur in real-life population dynamics remains unknown. In two dimensions, biodiversity prevails for small deviations from the non-transitive deterministic setting, due to the formation of spiral wave patterns. Although species evenness is impacted by the winning probabilities, the formation of spiral wave patterns leads to long coexistence times, underlining the importance of spatial segregation regardless of species evenness. Larger deviations from the non-transitive deterministic setting cut coexistence short, as no long-lived spatial patterns are able to form.

In conclusion, our work shows that non-transitive competition can still produce long coexistence times when the deterministic assumption is relaxed. As such, theoretical results obtained using the deterministic assumption may still hold in a more realistic setting. Also, ecosystems for which empirical studies measured competitive abilities that constitute a non-transitive non-deterministic competition network may be valid candidates for experimental research on the relation between non-transitivity and biodiversity, despite the competition network being non-deterministic. Exploratory studies, using synthetic organisms, that relate non-transitive competition to spatial segregation and pattern formation have already been conducted. ${ }^{37,42}$

In all spatial dimensions, a "survival of the strongest" law can be discerned: the species that has the highest probability of defeating its competitors has the best odds to become the sole survivor. Although this result seems to contradict the "survival of the weakest" law proposed in Ref. 30, we argue that this is due to the interpretation of the reaction rate constants associated with competition events in the Lotka-Volterra model used in Ref. 30, where it might be less confusing to name the species with the lowest reaction rate constant "the least aggressive," rather than "the weakest."

In Ref. 30 it is claimed that, since evolution would not head in a direction where a species becomes "weaker" such that it can outcompete its adversaries, non-transitive competition, as modelled through Lotka-Volterra dynamics, is evolutionarily stable. In our framework, the evolutionary stability of the ecosystem is less clear, due to the sensitive dependence of coexistence times on the winning probabilities. When all winning probabilities are approximately equal, coexistence times are long, and a weaker species may have the time to catch-up with its competitors through evolution. However, when winning probabilities become less symmetric, and one of the species thus is significantly stronger, coexistence times are short compared to evolutionary time scales, such that coexistence should be lost. Hence, in contrast to previous work, our model predicts that non-transitive competition does not necessarily imply an evolutionarily stable ecosystem, but rather that evolution could cause two species to go extinct, with the stronger species surviving.

\section{ACKNOWLEDGMENTS}

A. J. Daly acknowledges funding from a UGent-BOF GOA project "Assessing the biological capacity of ecosystem resilience" (Grant No. BOFGOA2017000601). The computational resources (Stevin Supercomputer Infrastructure) and services used in this work were provided by the VSC (Flemish Supercomputer Center), funded by the Ghent University, the Hercules Foundation, and the Flemish Government-Department EWI.

${ }^{1}$ G. Hardin, “The competitive exclusion principle," Science 131, 1292-1297 (1960).

${ }^{2}$ S. A. Levin, "Community equilibria and stability, and an extension of the competitive exclusion principle," Am. Nat. 104, 413-423 (1970).

${ }^{3}$ G. E. Hutchinson, "The paradox of the plankton," Am. Nat. 95, 137-145 (1961).

${ }^{4}$ P. Cermeño, I. G. Teixeira, M. Branco, F. G. Figueiras, and E. Marañón, "Sampling the limits of species richness in marine phytoplankton communities," J. Plankton Res. 36, 1135-1139 (2014).

${ }^{5}$ J. Huisman and F. J. Weissing, "Biodiversity of plankton by species oscillations and chaos," Nature 402, 407 (1999).

${ }^{6}$ L. Becks, F. M. Hilker, H. Malchow, K. Jürgens, and H. Arndt, "Experimental demonstration of chaos in a microbial food web," Nature 435, 1226 (2005).

${ }^{7}$ M. Scheffer and E. H. van Nes, "Self-organized similarity, the evolutionary emergence of groups of similar species," Proc. Natl. Acad. Sci. U.S.A. 103, 6230-6235 (2006).

${ }^{8}$ J. Tews, U. Brose, V. Grimm, K. Tielbörger, M. C. Wichmann, M. Schwager, and F. Jeltsch, "Animal species diversity driven by habitat heterogeneity/diversity: The importance of keystone structures," J. Biogeogr. 31, 79-92 (2004)

${ }^{9}$ U. Bastolla, M. A. Fortuna, A. Pascual-García, A. Ferrera, B. Luque, and J. Bascompte, "The architecture of mutualistic networks minimizes competition and increases biodiversity," Nature 458, 1018-1020 (2009).

${ }^{10}$ A.-M. Neutel, J. A. Heesterbeek, and P. C. de Ruiter, "Stability in real food webs: Weak links in long loops," Science 296, 1120-1123 (2002).

${ }^{11}$ J. M. Alcántara, M. Pulgar, and P. J. Rey, "Dissecting the role of transitivity and intransitivity on coexistence in competing species networks," Theor. Ecol. 10, 207-215 (2017).

${ }^{12}$ J. Knebel, T. Krüger, M. F. Weber, and E. Frey, "Coexistence and survival in conservative Lotka-Volterra networks," Phys. Rev. Lett. 110, 168106 (2013).

${ }^{13}$ H. Cheng, N. Yao, Z.-G. Huang, J. Park, Y. Do, and Y.-C. Lai, "Mesoscopic interactions and species coexistence in evolutionary game dynamics of cyclic competitions," Sci. Rep. 4, 7486 (2014). 
${ }^{14}$ J. Park, Y. Do, B. Jang, and Y.-C. Lai, "Emergence of unusual coexistence states in cyclic game systems," Sci. Rep. 7, 7465 (2017).

${ }^{15}$ R. M. May and W. J. Leonard, "Nonlinear aspects of competition between three species," SIAM J. Appl. Math. 29, 243-253 (1975).

${ }^{16}$ R. A. Laird and B. S. Schamp, "Does local competition increase the coexistence of species in intransitive networks?," Ecology 89, 237-247 (2008).

${ }^{17}$ R. A. Laird, "Population interaction structure and the coexistence of bacterial strains playing 'rock-paper-scissors'," Oikos 123, 472-480 (2014).

${ }^{18} \mathrm{~T}$. Reichenbach, M. Mobilia, and E. Frey, "Self-organization of mobile populations in cyclic competition," J. Theor. Biol. 254, 368-383 (2008).

${ }^{19}$ T. Reichenbach, M. Mobilia, and E. Frey, "Mobility promotes and jeopardizes biodiversity in rock-paper-scissors games," Nature 448, 1046-1049 (2007).

${ }^{20}$ P. Avelino, D. Bazeia, L. Losano, J. Menezes, and B. de Oliveira, "Interfaces with internal structures in generalized rock-paper-scissors models," Phys. Rev. E 89, 042710 (2014).

${ }^{21}$ L. Frachebourg, P. L. Krapivsky, and E. Ben-Naim, "Segregation in a onedimensional model of interacting species," Phys. Rev. Lett. 77, 21-25 (1996).

${ }^{22}$ L. Frachebourg, P. L. Krapivsky, and E. Ben-Naim, "Spatial organization in cyclic Lotka-Volterra systems," Phys. Rev. E 54, 6186-6200 (1996).

${ }^{23} \mathrm{~S}$. Rulands, T. Reichenbach, and E. Frey, "Threefold way to extinction in populations of cyclically competing species," J. Stat. Mech. Theory E 2011, L01003 (2011)

${ }^{24} \mathrm{~S}$. Venkat and M. Pleimling, "Mobility and asymmetry effects in onedimensional rock-paper-scissors games,” Phys. Rev. E 81, 021917 (2010).

${ }^{25}$ N. Mitarai, I. Gunnarson, B. N. Pedersen, C. A. Rosiek, and K. Sneppen, "Three is much more than two in coarsening dynamics of cyclic competitions," Phys. Rev. E 93, 042408 (2016).

${ }^{26} \mathrm{M}$. Peltomäki and M. Alava, "Three- and four-state rock-paper-scissors games with diffusion," Phys. Rev. E 78, 031906 (2008).

${ }^{27}$ J. Grilli, G. Barabás, M. J. Michalska-Smith, and S. Allesina, "Higher-order interactions stabilize dynamics in competitive network models," Nature 548, 210-213 (2017).

${ }^{28} \mathrm{Q}$. He, M. Mobilia, and U. C. Täuber, "Coexistence in the two-dimensional may-leonard model with random rates," Eur. Phys. J. B 82, 97-105 (2011).

${ }^{29} \mathrm{Q}$. He, M. Mobilia, and U. C. Täuber, "Spatial rock-paper-scissors models with inhomogeneous reaction rates," Phys. Rev. E 82, 051909 (2010).

${ }^{30}$ M. Frean and E. R. Abraham, "Rock-scissors-paper and the survival of the weakest,” Proc. R. Soc. Lond. B Bio. 268, 1323-1327 (2001).

${ }^{31}$ J. Park, Y. Do, Z.-G. Huang, and Y.-C. Lai, "Persistent coexistence of cyclically competing species in spatially extended ecosystems," Chaos $\mathbf{2 3}$, 023128 (2013).

${ }^{32} \mathrm{~B}$. Kim and J. Park, "Basins of distinct asymptotic states in the cyclically competing mobile five species game," Chaos 27, 103117 (2017).

${ }^{33}$ J. Park, "Biodiversity in the cyclic competition system of three species according to the emergence of mutant species," Chaos 28, 053111 (2018).

${ }^{34}$ R. Yang, W.-X. Wang, Y.-C. Lai, and C. Grebogi, "Role of intraspecific competition in the coexistence of mobile populations in spatially extended ecosystems," Chaos 20, 023113 (2010).

${ }^{35}$ R. Durrett and S. Levin, "Spatial aspects of interspecific competition," Theor. Popul. Biol. 53, 30-43 (1998).

${ }^{36}$ R. Durrett and S. Levin, "Allelopathy in spatially distributed populations," J. Theor. Biol. 185, 165-171 (1997).

${ }^{37}$ B. Kerr, M. Riley, M. W. Feldman, and B. J. M. Bohannan, "Local dispersal promotes biodiversity in a real-life game of rock-paper-scissors," Nature 418, 171-174 (2002)

${ }^{38}$ T. L. Czárán, R. F. Hoekstra, and L. Pagie, "Chemical warfare between microbes promotes biodiversity,” Proc. Natl. Acad. Sci. U.S.A. 99, 786-790 (2002)

${ }^{39}$ J. Jackson and L. Buss, "Alleopathy and spatial competition among coral reef invertebrates," Proc. Natl. Acad. Sci. U.S.A. 72, 5160-5163 (1975).

${ }^{40} \mathrm{~B}$. Sinervo and C. M. Lively, "The rock-scissors-paper game and the evolution of alternative male strategies," Nature 380, 240-243 (1996).

${ }^{41}$ O. Gilg, I. Hanski, and B. Sittler, "Cyclic dynamics in a simple vertebrate predator-prey community," Science 302, 866-868 (2003).

${ }^{42}$ F. J. Hol, M. J. Voges, C. Dekker, and J. E. Keymer, "Nutrient-responsive regulation determines biodiversity in a colicin-mediated bacterial community," BMC Biol. 12, 1 (2014).

${ }^{43}$ J. M. Levine, J. Bascompte, P. B. Adler, and S. Allesina, "Beyond pairwise mechanisms of species coexistence in complex communities," Nature 546, 56-64 (2017).
${ }^{44}$ P. Funston, M. Mills, and H. Biggs, "Factors affecting the hunting success of male and female lions in the Kruger National Park," J. Zool. 253, 419-431 (2001).

${ }^{45}$ E. Litchman and C. A. Klausmeier, "Competition of phytoplankton under fluctuating light,” Am. Nat. 157, 170-187 (2001).

${ }^{46} \mathrm{~S}$. P. Hubbell, "Neutral theory in community ecology and the hypothesis of functional equivalence," Funct. Ecol. 19, 166-172 (2005).

${ }^{47}$ J. D. Fridley and J. P. Grime, "Community and ecosystem effects of intraspecific genetic diversity in grassland microcosms of varying species diversity," Ecology 91, 2272-2283 (2010).

${ }^{48}$ S. P. Hart, S. J. Schreiber, and J. M. Levine, "How variation between individuals affects species coexistence," Ecol. Lett. 19, 825-838 (2016).

${ }^{49}$ B. De Baets, H. De Meyer, and B. De Schuymer, "Cyclic evaluation of transitivity of reciprocal relations," Soc. Choice Welfare 26, 217-238 (2006).

${ }^{50}$ J. R. Nahum, B. N. Harding, and B. Kerr, "Evolution of restraint in a structured rock-paper-scissors community," Proc. Natl. Acad. Sci. U.S.A. 108, 10831-10838 (2011).

${ }^{51}$ J. Männik, R. Driessen, P. Galajda, J. E. Keymer, and C. Dekker, "Bacterial growth and motility in sub-micron constrictions," Proc. Natl. Acad. Sci. U.S.A. 106, 14861-14866 (2009).

${ }^{52}$ R. Durrett and S. Levin, "The importance of being discrete (and spatial)," Theor. Popul. Biol. 46, 363-394 (1994).

${ }^{53}$ T. M. Liggett, Interacting Particle Systems (Springer Science and Business Media, Berlin, 2012).

${ }^{54}$ E. Frey, "Evolutionary game theory: Theoretical concepts and applications to microbial communities," Physica A 389, 4265-4298 (2010).

${ }^{55}$ N. vanKampen, Stochastic Processes in Physics and Chemistry (Elsevier, Amsterdam, 2007), p. 420.

${ }^{56}$ M. L. Zeeman, "Hopf bifurcations in competitive three-dimensional LotkaVolterra systems," Dynam. Stabil. Syst. 8, 189-216 (1993).

${ }^{57}$ M. W. Hirsch, "Systems of differential equations that are competitive or cooperative. III: Competing species," Nonlinearity 1, 51-71 (1988).

${ }^{58}$ M. L. Zeeman and P. van den Driessche, "Three-dimensional competitive Lotka-Volterra systems with no periodic orbits," SIAM J. Appl. Math. 58, 227-234 (1998).

${ }^{59}$ M. P. Hassell, H. N. Comins, and R. M. May, "Species coexistence and self-organizing spatial dynamics," Nature 370, 290-292 (1994).

${ }^{60}$ A.-K. Kassam and L. N. Trefethen, "Fourth-order time-stepping for stiff PDEs," SIAM J. Sci. Comput. 26, 1214-1233 (2005).

${ }^{61}$ P. Chesson, "Mechanisms of maintenance of species diversity," Annu. Rev. Ecol. Syst. 31, 343-366 (2000).

${ }^{62}$ J. C. D. Terry, R. J. Morris, and M. B. Bonsall, "Trophic interaction modifications: An empirical and theoretical framework," Nature 20, 1219-1230 (2017).

${ }^{63}$ J. M. Levine, J. Bascompte, P. B. Adler, and S. Allesina, "Beyond pairwise mechanisms of species coexistence in complex communities," Nature 546, 56-64 (2017)

${ }^{64}$ S. H. Roxburgh and J. B. S. Wilson, "Stability and coexistence in a lawn community: Mathematical prediction of stability using a community matrix with parameters derived from competition experiments," Oikos 88, 395-408 (2000).

${ }^{65}$ F. J. Ayala, M. E. Gilpin, and J. G. Ehrenfeld, "Competition between species: Theoretical models and experimental tests," Theor. Popul. Biol. 4, 331-356 (1973).

${ }^{66}$ A. M. Siepielski, K. L. Hung, E. E. B. Bein, and M. A. McPeek, "Experimental evidence for neutral community dynamics governing an insect assemblage," Ecology 91, 847-857 (2010).

${ }^{67}$ M. J. A. Christianen, T. van der Heide, S. J. Holthuijsen, K. J. van der Reijden, A. C. W. Borst, and H. Olff, "Biodiversity and food web indicators of community recovery in intertidal shellfish reefs," Biol. Conserv. 213, 317-324 (2017).

${ }^{68}$ J. McGrady-Steed, P. M. Harris, and P. J. Morin, "Biodiversity regulates ecosystem predictability," Nature 390, 162-165 (1997).

${ }^{69}$ S. Prasad, P. Manasa, S. Buddhi, S. M. Singh, and S. Shivaji, "Antagonistic interaction networks among bacteria from a cold soil environment," FEMS Microbiol. Ecol. 78, 376-385 (2011).

${ }^{70}$ A. J. Daly, J. M. Baetens, and B. De Baets, "The impact of initial evenness on biodiversity maintenance for a four-species in silico bacterial community,” J. Theor. Biol. 387, 189-205 (2015).

${ }^{71}$ J. Vukov, A. Szolnoki, and G. Szabó, "Diverging fluctuations in a spatial five-species cyclic dominance game," Phys. Rev. E 88, 022123 (2013). 